\title{
Structure and lattice dynamics of the wide band gap semiconductors $\mathrm{MgSiN}_{2}$ and $\mathrm{MgGeN}_{2}$
}

M. Råsander, J. B. Quirk, T. Wang, S. Mathew, R. Davies, R. G. Palgrave, and M. A. Moram

Citation: Journal of Applied Physics 122, 085705 (2017);

View online: https://doi.org/10.1063/1.4985775

View Table of Contents: http://aip.scitation.org/toc/jap/122/8

Published by the American Institute of Physics

\section{Articles you may be interested in}

Structural and electrical properties of single crystalline $\mathrm{SrZrO}_{3}$ epitaxially grown on $\mathrm{Ge}(001)$

Journal of Applied Physics 122, 084102 (2017); 10.1063/1.5000142

Enhanced room temperature ferromagnetism in $\mathrm{Ni}$ doped $\mathrm{SnO}_{2}$ nanoparticles: A comprehensive study Journal of Applied Physics 122, 083906 (2017); 10.1063/1.4999830

Electronic band structures and excitonic properties of delafossites: A GW-BSE study Journal of Applied Physics 122, 085104 (2017); 10.1063/1.4991913

First-principles and molecular dynamics study of thermoelectric transport properties of N-type silicon-based superlattice-nanocrystalline heterostructures

Journal of Applied Physics 122, 085105 (2017); 10.1063/1.5000356

Influence of the heterostructure design on the optical properties of $\mathrm{GaN}$ and $\mathrm{Al}_{0.1} \mathrm{Ga}_{0.9} \mathrm{~N}$ quantum dots for ultraviolet emission

Journal of Applied Physics 122, 085706 (2017); 10.1063/1.5000238

Effects of thickness ratio of InGaN to GaN in superlattice strain relief layer on the optoelectrical properties of InGaN-based green LEDs grown on Si substrates

Journal of Applied Physics 122, 084504 (2017); 10.1063/1.5000134

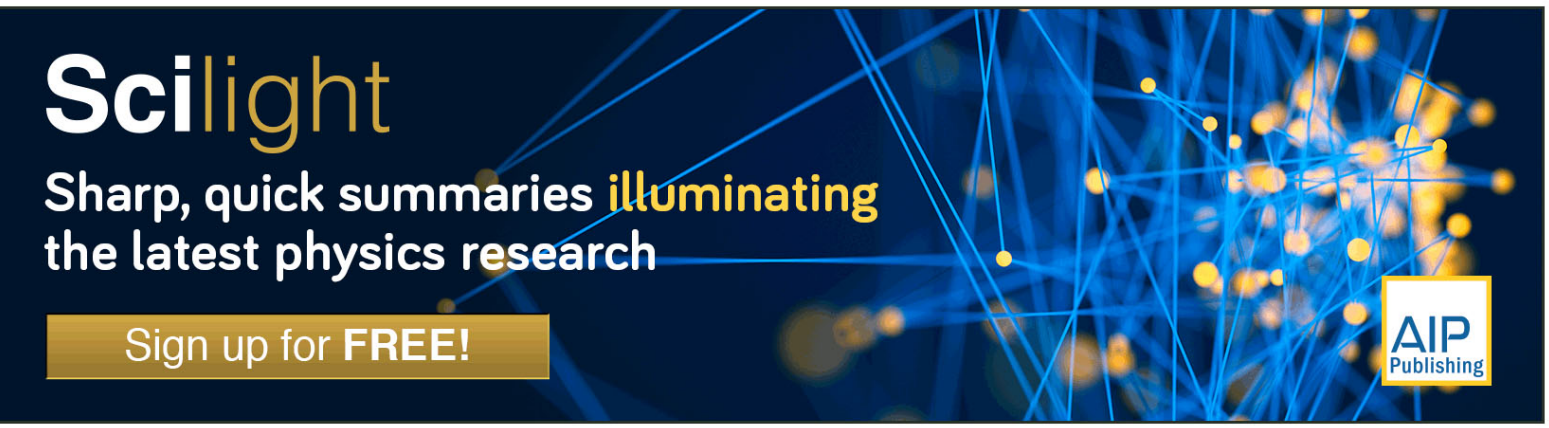




\title{
Structure and lattice dynamics of the wide band gap semiconductors $\mathrm{MgSiN}_{2}$ and $\mathrm{MgGeN}_{2}$
}

\author{
M. Råsander, ${ }^{1, a)}$ J. B. Quirk, ${ }^{1}$ T. Wang, ${ }^{1}$ S. Mathew,${ }^{2}$ R. Davies, ${ }^{1}$ R. G. Palgrave,${ }^{2}$ \\ and M. A. Moram ${ }^{1}$ \\ ${ }^{1}$ Department of Materials, Imperial College London, Exhibition Road, SW7 2AZ London, United Kingdom \\ ${ }^{2}$ Department of Chemistry, University College London, Gordon Street, WC1H OAJ London, United Kingdom
}

(Received 16 May 2017; accepted 11 August 2017; published online 30 August 2017)

\begin{abstract}
We have determined the structural and lattice dynamical properties of the orthorhombic, wide band gap semiconductors $\mathrm{MgSiN}_{2}$ and $\mathrm{MgGeN}_{2}$ using density functional theory. In addition, we present the structural properties and Raman spectra of $\mathrm{MgSiN}_{2}$ powder. The structural properties and lattice dynamics of the orthorhombic systems are compared to those of wurtzite AlN. We find clear differences in the lattice dynamics between $\mathrm{MgSiN}_{2}, \mathrm{MgGeN}_{2}$ and $\mathrm{AlN}$, for example, we find that the highest phonon frequency in $\mathrm{MgSiN}_{2}$ is about $100 \mathrm{~cm}^{-1}$ higher than the highest frequency in $\mathrm{AlN}$, and that $\mathrm{MgGeN}_{2}$ is much softer. We also provide the Born effective charge tensors and dielectric tensors of $\mathrm{MgSiN}_{2}, \mathrm{MgGeN}_{2}$ and $\mathrm{AlN}$. Phonon related thermodynamic properties, such as the heat capacity and the entropy, have also been evaluated and are found to be in very good agreement with available experimental results. Published by AIP Publishing.
\end{abstract}

[http://dx.doi.org/10.1063/1.4985775]

\section{INTRODUCTION}

Group III-nitride semiconductors AlN, GaN and $\mathrm{InN}$ are widely used in optoelectronic and high power electronic devices. ${ }^{1-3}$ AlN and its alloys are also widely used in energy harvesting devices and RF applications. ${ }^{4}$ However, improved efficiencies are required for III-nitride-based ultraviolet (UV) light emitting diodes, ${ }^{5}$ solar cells ${ }^{6}$ and energy harvesting devices. ${ }^{4}$ Group II-IV nitride semiconductors are of growing interest in this regard, as their bonding and crystal structures are related to those of III-nitrides, but they offer different combinations of band gaps and lattice parameters, opening up additional possibilities for device design. ${ }^{7,8}$ For example, Zn-based II-IV nitrides are of current interest for solar cells, ${ }^{7,8}$ whereas wide band gap II-IV nitrides, such as $\mathrm{MgSiN}_{2}$, may find applications as part of UV optoelectronic devices. $^{9-11}$

$\mathrm{MgSiN}_{2}$ and $\mathrm{MgGeN}_{2}$ have been found to possess a wurtzite-derived orthorhombic crystal structure which belongs to the Pna 2 space group (No. 33). ${ }^{12}$ The band gap of $\mathrm{MgSiN}_{2}$ has been investigated by both theory ${ }^{9-11,13-15}$ and experiment, $9,15,16$ and it has been found that the band gap in $\mathrm{MgSiN}_{2}$ is indirect with a similar size as the band gap in wurtzite AIN. ${ }^{9,11,15}$ A recent study employing the GW approximation has established that the band gap decreases from $5.85 \mathrm{eV}$ in $\mathrm{MgSiN}_{2}$ to $5.14 \mathrm{eV}$ in $\mathrm{MgGeN}_{2}$ to $3.43 \mathrm{eV}$ in $\mathrm{MgSnN}_{2}$ when the Group IV element is substituted from $\mathrm{Si}$ to Ge to Sn. ${ }^{11}$ It was also found that the band gaps in both $\mathrm{MgGeN}_{2}$ and $\mathrm{MgSnN}_{2}$ are direct, in contrast to the indirect gap in $\mathrm{MgSiN}_{2}$. A similar trend is also observed for $\mathrm{Zn}-\mathrm{IV}$ $\mathrm{N}_{2}$ nitride phases, ${ }^{7,8}$ however, the sizes of the band gaps are smaller in these Zn-based systems than in the equivalent $\mathrm{Mg}$-based II-IV nitrides. In addition to having a large band

${ }^{\text {a)} E l e c t r o n i c ~ m a i l: ~ m . r a s a n d e r @ i m p e r i a l . a c . u k ~}$ gap, $\mathrm{MgSiN}_{2}$ has been found to possess a small anisotropic thermal expansion, ${ }^{17}$ and a thermal conductivity of $28 \mathrm{~W} /$ $\mathrm{mK}$ was obtained for the powder at $300 \mathrm{~K} .{ }^{18}$ These properties together with the great lattice matching between $\mathrm{MgSiN}_{2}$ and $\mathrm{AlN}^{9}$ suggest that $\mathrm{MgSiN}_{2}$ could be used for short wavelength or high power electronic devices where heat transport away from the active region of the device is highly desirable.

Even though the understanding of the electronic properties of the Mg-IV- $\mathrm{N}_{2}$ systems is increasing, not much is known regarding the lattice dynamics and related properties. Apart from experimental studies of thermal expansion, ${ }^{17}$ thermal conductivity ${ }^{18}$ and heat capacity ${ }^{19}$ of $\mathrm{MgSiN}_{2}$, there are two recent theoretical studies focusing on phonon related properties: Arab et al. ${ }^{20}$ used a simple quasi-harmonic Debye model to evaluate the thermodynamic properties of $\mathrm{MgSiN}_{2}$, while Pramchu et al. ${ }^{21}$ used density functional perturbation theory in combination with the local density approximation to evaluate the phonon dispersions of both $\mathrm{MgSiN}_{2}$ and $\mathrm{MgGeN}_{2}$. However, neither of these studies provide a connection between the lattice dynamics and the thermodynamic properties of these compounds. In this study, we present structural as well as phonon-related properties, such as phonon dispersions, phonon density of states, Helmholtz free energy and heat capacity, of $\mathrm{MgSiN}_{2}$ and $\mathrm{MgGeN}_{2}$ to provide a connection between the structure, the lattice dynamics and the thermodynamic properties of these systems. The bulk of the results presented here is obtained using calculations based on density functional theory. However, we also provide experimental structural properties and Raman spectra of $\mathrm{MgSiN}_{2}$ powder. In order to facilitate greater understanding, the properties established for $\mathrm{MgSiN}_{2}$ and $\mathrm{MgGeN}_{2}$ will be compared with those of wurtzite AlN, which is the starting point for obtaining the crystal structure of $\mathrm{MgSiN}_{2}$. We note that both $\mathrm{MgSiN}_{2}$ and $\mathrm{MgGeN}_{2}$ have been produced in powder form previously, ${ }^{12}$ and, 
considering the size of the band gaps, ${ }^{11}$ are of interest in UV-optoelectronic applications.

\section{METHOD}

Density functional calculations have been performed using the projector augmented wave (PAW) method ${ }^{22}$ as implemented in the Vienna $a b$ initio simulation package (VASP). ${ }^{23,24} \mathrm{We}$ have used the generalized gradient approximation of Perdew, Burke and Ernzerhof $(\mathrm{PBE})^{25}$ for the exchange-correlation energy functional. The plane wave energy cut-off was set to $800 \mathrm{eV}$, and we have used $\Gamma$-centered k-point meshes with the smallest allowed spacing between k-points of $0.1 \AA^{-1}$. The atomic positions and the simulation cell shapes were relaxed until the Hellmann-Feynman forces acting on atoms were smaller than $0.0001 \mathrm{eV} / \AA$ A. Furthermore, we have used the standard core-valence partitioning for $\mathrm{Mg}$ (with a $3 \mathrm{~s}^{2}$ valence), $\mathrm{Si}$ (with a $3 s^{2} 3 p^{2}$ valence), $\mathrm{Al}$ (with a $3 s^{2} 3 p^{1}$ valence) and $\mathrm{N}$ (with a $2 s^{2} 2 p^{3}$ valence). In the case of $\mathrm{Ge}$, we have used both the four $4 \mathrm{~s}$ and $4 \mathrm{p}$ electrons and the semi-core $3 \mathrm{~d}$ electrons as valence states.

The evaluation of phonon related properties has been done using the finite displacement method as it is implemented in the Phonopy code. ${ }^{26,27}$ For $\mathrm{MgSiN}_{2}$ and $\mathrm{MgGeN}_{2}$, we have used supercells based on a $3 \times 3 \times 3$ repetition of the primitive orthorhombic unit cells containing 432 atoms. In the case of AlN, we have used a $5 \times 5 \times 4$ repetition of the primitive wurtzite unit cell with 400 atoms in the supercell. The phonon density of states and the related properties were obtained using a $24 \times 24 \times 24$ q-point mesh for $\mathrm{MgSiN}_{2}$ and $\mathrm{MgGeN}_{2}$ and a $48 \times 48 \times 48$ q-point mesh for AlN.

In polar crystals, a macroscopic electric field is induced by the collective displacement of the ions at $\boldsymbol{q}=0$. This electric field affects the longitudinal optical (LO) modes and not the transverse optical (TO) modes, and therefore gives rise to a LO-TO splitting of the modes when approaching the $\Gamma$-point. To account for this effect, a non-analytical correction to the dynamical matrix of the form

$$
D_{\alpha j, \beta j^{\prime}}^{N A}(\boldsymbol{q} \rightarrow 0)=\frac{4 \pi}{\Omega_{0} \sqrt{m_{j} m_{j^{\prime}}}} \frac{\left[\sum_{\gamma} q_{\gamma} \boldsymbol{Z}_{j, \gamma \gamma \alpha}^{*}\right]\left[\sum_{\gamma^{\prime}} q_{\gamma^{\prime}} \boldsymbol{Z}_{j^{\prime}, \gamma^{\prime} \beta}^{*}\right]}{\sum_{\alpha \beta} q_{\alpha} \boldsymbol{\varepsilon}_{\alpha \beta}^{\infty} q_{\beta}}
$$

is added. ${ }^{28-31}$ Here, $\boldsymbol{\varepsilon}^{\infty}$ is the high-frequency dielectric tensor and $\boldsymbol{Z}_{s}^{*}$ is the Born effective charge tensor for the atom $s$. The dielectric tensors and Born effective charge tensors of $\mathrm{MgSiN}_{2}, \mathrm{MgGeN}_{2}$ and AlN were calculated using density functional perturbation theory, ${ }^{32}$ as outlined by Gajdoš et al. ${ }^{33}$

In addition, $\mathrm{MgSiN}_{2}$ powders were synthesised by firing stoichiometric $\mathrm{Mg}$ and $\mathrm{Si}_{3} \mathrm{~N}_{4}$ powders in a horizontal tube furnace for $16 \mathrm{~h}$ at $1000^{\circ} \mathrm{C}$ at a heating rate of $10^{\circ} \mathrm{C} / \mathrm{min}$ under a flowing $\mathrm{N}_{2}(700 \mathrm{sccm})$ atmosphere in a Mo boat, prior to cooling at $5{ }^{\circ} \mathrm{C} / \mathrm{min}$. To verify the crystal structure, powder $\mathrm{X}$ ray diffraction (XRD) was performed over the range $20^{\circ}-120^{\circ}$ $2 \theta$, using $\mathrm{Cu}-\mathrm{K} \alpha_{1}$ radiation. Furthermore, Raman measurements were collected using a LabRAM HR Evolution spectrometer (Horiba Scientific) using a laser wavelength of $532 \mathrm{~nm}$, an objective lens of $\times 10$, and a grating of 600 lines/ $\mathrm{mm}$. Spectra were recorded over the wavenumber range 100 to $1250 \mathrm{~cm}^{-1}$ with an acquisition time of $100 \mathrm{~s}$.

\section{STRUCTURAL PROPERTIES}

The ordered $\mathrm{MgSiN}_{2}$ and $\mathrm{MgGeN}_{2}$ structures can be derived from the AlN structure by substituting one $\mathrm{Mg}$ and one $\mathrm{Si}$ (or $\mathrm{Ge}$ ) atom for every two $\mathrm{Al}$ atoms. The structures have been found to be orthorhombic and belong to the Pna2 $2_{1}$ space group (No. 33), ${ }^{12,17}$ where all atoms occupy the $4 a$ Wyckoff crystal positions, and the unit cell, therefore, contains 4 formula units of $\mathrm{MgSiN}_{2}$ or $\mathrm{MgGeN}_{2}$. The crystal structure is shown in Fig. 1, and the structural parameters are presented in Table I for $\mathrm{MgSiN}_{2}$ and in Table II for $\mathrm{MgGeN}_{2}$. We note that in this structure, there are two different nitrogen positions, labelled $\mathrm{N}(1)$ and $\mathrm{N}(2)$. The $\mathrm{N}(1)$ positions are approximately positioned above the $\mathrm{Si}$ (or $\mathrm{Ge}$ ) positions along the $c$-axis, while the $\mathrm{N}(2)$ positions are approximately positioned above the $\mathrm{Mg}$ positions. In order to satisfy local charge neutrality, each of the $\mathrm{N}(1)$ and $\mathrm{N}(2)$ positions has two $\mathrm{Mg}$ and two $\mathrm{Si}$ (or $\mathrm{Ge}$ ) atoms as the nearest neighbours. Correspondingly, each of the $\mathrm{Mg}$ and $\mathrm{Si}$ (or $\mathrm{Ge}$ ) positions has two $\mathrm{N}(1)$ and two $\mathrm{N}(2)$ positions as the nearest neighbours.

In Fig. 2, we show the measured XRD pattern of $\mathrm{MgSiN}_{2}$, which shows that the structure is orthorhombic and belongs to the Pna2 ${ }_{1}$ space group. We also find peaks related to pure $\mathrm{Si}$ and $\mathrm{MgO}$ impurities in the sample. The corresponding $\mathrm{MgSiN}_{2}$ lattice constants are shown in Table I. The measured lattice constants are in very good agreement with previously measured lattice constants. ${ }^{17}$

As shown in Tables I and II, we find that the PBE calculations overestimate the lattice constants compared to available experiments, with a maximum deviation of $0.9 \%$ for $\mathrm{MgSiN}_{2}$ and $1.1 \%$ for $\mathrm{MgGeN}_{2}$, which is to be expected using the PBE approximation for these types of systems. ${ }^{34}$ The calculated structural parameters are also in agreement

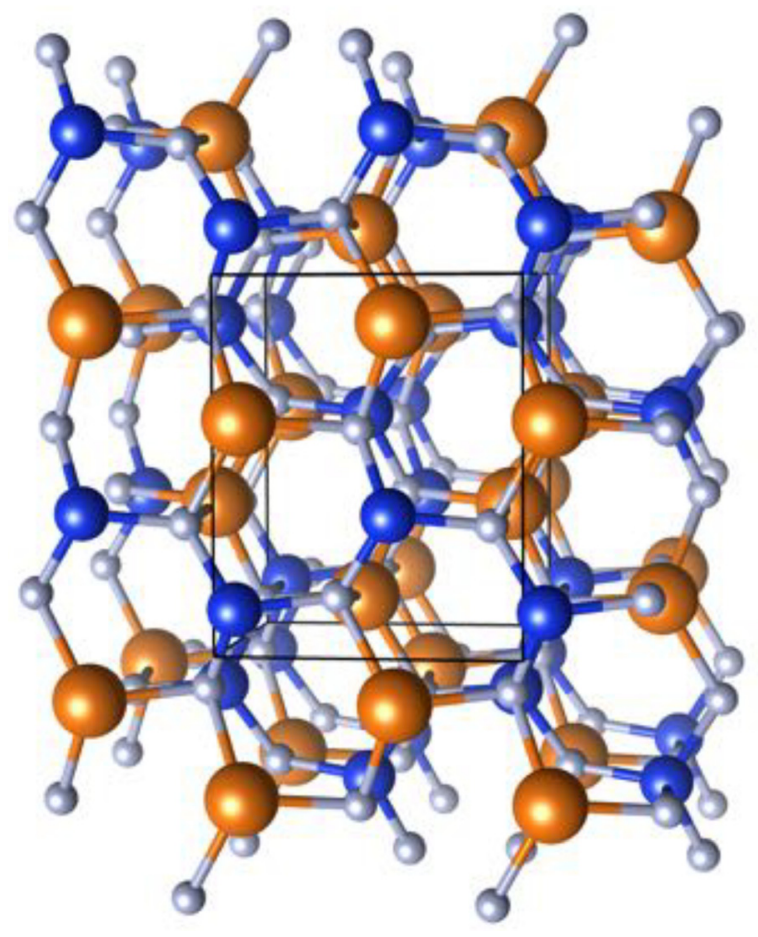

FIG. 1. The orthorhombic crystal structure (space group Pna2 $2_{1}$ ) of $\mathrm{MgSiN}_{2}$ and $\mathrm{MgGeN}_{2} . \mathrm{Mg}$ is represented by the large bronze colored spheres, $\mathrm{Si}$ (or Ge) is represented by blue spheres, while $\mathrm{N}$ is represented by small grey spheres. 
TABLE I. Lattice constants and crystallographic coordinates $(x, y, z)$ of orthorhombic $\mathrm{MgSiN}_{2}$. The numbers in parenthesis are the deviation from the experimental value.

\begin{tabular}{|c|c|c|c|}
\hline $\mathrm{XC}$ & $a(\AA)$ & $b(\AA)$ & $c(\AA)$ \\
\hline PBE & $5.311(0.8 \%)$ & $6.495(0.4 \%)$ & $5.028(0.9 \%)$ \\
\hline Expt. $(T=300 \mathrm{~K})$ & 5.314 & 6.466 & 4.975 \\
\hline \multirow[t]{3}{*}{ Expt. $(T=10 \mathrm{~K})^{17}$} & $5.27078(5)$ & $6.46916(7)$ & $4.98401(5)$ \\
\hline & \multicolumn{3}{|c|}{ PBE } \\
\hline & $x$ & $y$ & $z$ \\
\hline $\mathrm{Mg}$ & 0.0849 & 0.6228 & 0.9886 \\
\hline $\mathrm{Si}$ & 0.0700 & 0.1254 & 0.0000 \\
\hline $\mathrm{N}(1)$ & 0.0485 & 0.0957 & 0.3471 \\
\hline \multirow[t]{3}{*}{$\mathrm{N}(2)$} & 0.1093 & 0.6553 & 0.4102 \\
\hline & \multicolumn{3}{|c|}{ Expt. $(T=10 \mathrm{~K})^{17}$} \\
\hline & $x$ & $y$ & $z$ \\
\hline $\mathrm{Mg}$ & $0.08448(34)$ & $0.62255(30)$ & $0.9866(5)$ \\
\hline $\mathrm{Si}$ & $0.0693(5)$ & $0.1249(4)$ & 0.0000 \\
\hline $\mathrm{N}(1)$ & $0.04855(17)$ & $0.09562(15)$ & $0.3472(4)$ \\
\hline $\mathrm{N}(2)$ & $0.10859(18)$ & $0.65527(14)$ & $0.4102(4)$ \\
\hline
\end{tabular}

with previous results obtained using local and semi-local approximations for the exchange-correlation energy functional. ${ }^{11,13,14} \mathrm{We}$ note that $\mathrm{MgGeN}_{2}$ has a unit cell volume of $192.96 \AA^{3}$ (12.06 $\AA^{3}$ /atom), while the corresponding volume of $\mathrm{MgSiN}_{2}$ is $173.44 \AA^{3}$ (10.84 $\AA^{3}$ /atom). $\mathrm{MgGeN}_{2}$ is, therefore, a larger crystal than $\mathrm{MgSiN}_{2}$ and, due to the larger mass of Ge compared to $\mathrm{Si}$, the mass density of $\mathrm{MgGeN}_{2}$ is also larger than in $\mathrm{MgSiN}_{2}$. In the case of wurtzite $\mathrm{AlN}$, the calculated structural parameters are $a=3.127 \AA$, $c=5.013 \AA$ and $u=0.382$, which are in good agreement with experiment. When comparing the volume per atom of $\mathrm{MgSiN}_{2}$ with that of $\mathrm{AlN}$, we find that the volume of $\mathrm{MgSiN}_{2}$ is

TABLE II. Lattice constants and crystallographic coordinates $(x, y, z)$ of orthorhombic $\mathrm{MgGeN}_{2}$. The numbers in parenthesis are the deviation from the experimental value.

\begin{tabular}{lccc}
\hline \hline $\mathrm{XC}$ & $a(\AA)$ & $b(\AA)$ & $c(\AA)$ \\
\hline PBE & $5.549(1.0 \%)$ & $6.658(0.7 \%)$ & $5.223(1.1 \%)$ \\
Expt. [12] & 5.494 & 6.611 & 5.166 \\
& & PBE & \\
& & &
\end{tabular}

\begin{tabular}{lccc} 
& $x$ & $y$ & $z$ \\
\cline { 2 - 4 } $\mathrm{Mg}$ & 0.0851 & 0.6234 & 0.9929 \\
$\mathrm{Ge}$ & 0.0736 & 0.1258 & 0.0000 \\
$\mathrm{~N}(1)$ & 0.0604 & 0.1073 & 0.3600 \\
$\mathrm{~N}(2)$ & 0.1002 & 0.6431 & 0.3975 \\
& & Expt. $^{12}$ & \\
& & $y$ & $z$ \\
\hline $\mathrm{Mg}$ & 0.083 & 0.625 & 0.000 \\
$\mathrm{Ge}$ & 0.083 & 0.125 & 0.000 \\
$\mathrm{~N}(1)$ & 0.083 & 0.125 & 0.380 \\
$\mathrm{~N}(2)$ & 0.083 & 0.625 & 0.400 \\
\hline \hline
\end{tabular}

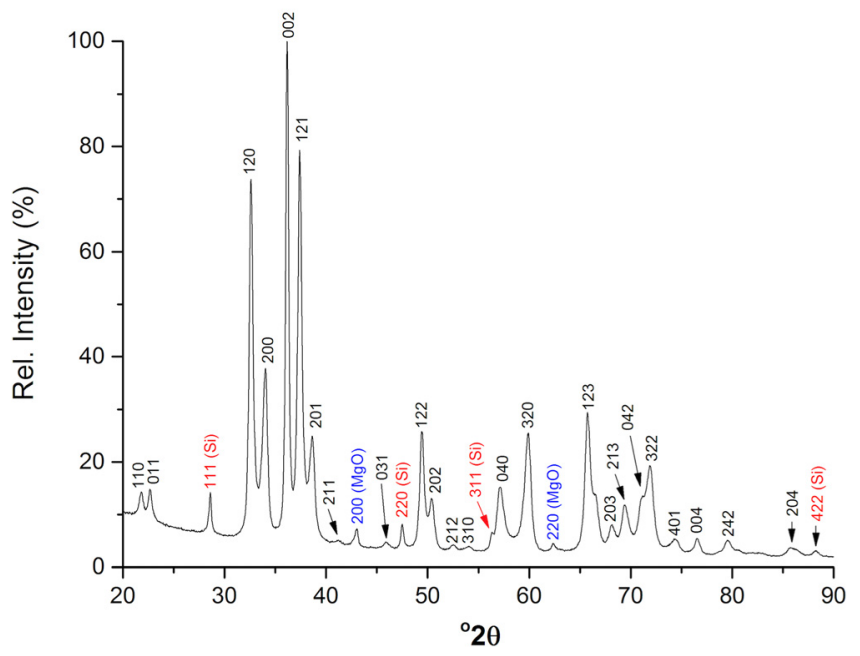

FIG. 2. Measured X-ray diffractogram of $\mathrm{MgSiN}_{2}$ powder, indexed as the ordered orthorhombic structure belonging to the Pna $2_{1}$ space group. Peaks corresponding to the $\mathrm{MgO}$ and $\mathrm{Si}$ impurity phases are highlighted using blue and red colours, respectively.

slightly larger at $10.84 \AA^{3} /$ atom compared to $10.61 \AA^{3} /$ atom in the case of AlN, which leads to the mass density being larger in $\mathrm{AlN}$ than in $\mathrm{MgSiN}_{2}$.

In $\mathrm{MgSiN}_{2}$ and $\mathrm{MgGeN}_{2}$, all atoms are locally found in a distorted tetrahedral environment, which has been discussed in detail by Bruls et al. ${ }^{17}$ For $\mathrm{MgSiN}_{2}$, we obtain average $\mathrm{Mg}$ $\mathrm{N}$ and Si-N binding distances of $2.11 \AA$ and $1.76 \AA$, respectively, which are slightly larger than $2.09 \AA$ and $1.75 \AA$ obtained by Bruls et al. ${ }^{17}$ In the case of $\mathrm{MgGeN}_{2}$, we obtain $\mathrm{Mg}-\mathrm{N}$ and Ge-N binding distances of $2.10 \AA$ and $1.89 \AA$, respectively, i.e., the average $\mathrm{Mg}-\mathrm{N}$ binding distance in $\mathrm{MgGeN}_{2}$ is slightly shorter than in $\mathrm{MgSiN}_{2}$ whereas the average IV-N binding distance is significantly longer in $\mathrm{MgGeN}_{2}$. In AlN, the average Al-N binding distance is $1.90 \AA$. If we average over the $\mathrm{Mg}-\mathrm{N}$ and $\mathrm{Si}-\mathrm{N}$ binding distances in $\mathrm{MgSiN}_{2}$, the resulting average binding distance is $1.94 \AA$, which is slightly larger than the binding distance in AlN, and if we average over the $\mathrm{Mg}-\mathrm{N}$ and $\mathrm{Ge}-\mathrm{N}$ binding distances we find the average binding distance in $\mathrm{MgGeN}_{2}$ to be $2.00 \AA$.

The orthorhombic structure discussed here is derived from the wurtzite crystal structure by transforming the lattice vectors in the $x y$-plane as $\boldsymbol{a}=\boldsymbol{a}_{1}+2 \boldsymbol{a}_{2}$ and $\boldsymbol{b}=2 \boldsymbol{a}_{1}$, while keeping the $\boldsymbol{c}$ lattice vector the same. Here, $\boldsymbol{a}_{1}$ and $\boldsymbol{a}_{2}$ are the in-plane lattice vectors of the wurtzite crystal structure, while $\boldsymbol{a}$ and $\boldsymbol{b}$ are the in-plane lattice constants in the orthorhombic structure. The relation between the in-plane lattice constant in wurtzite, $a_{w}$, and the $a$ and $b$ lattice constants of the orthorhombic structure is therefore $a_{w} \approx a / \sqrt{3} \approx b / 2$. Note that if pure AlN was calculated using the orthorhombic crystal structure $a_{w}=a / \sqrt{3}=b / 2$. The change from $=$ to $\approx$ is due to relaxations in the $\mathrm{MgSiN}_{2}$ and $\mathrm{MgGeN}_{2}$ systems. It is therefore possible to evaluate an average wurtzite-like lattice constant for the orthorhombic structures as $\bar{a}_{w}=(a / \sqrt{3}$ $+b / 2) / 2$. For $\mathrm{MgSiN}_{2}$, the wurtzite-like lattice constant is $3.157 \AA$, which is only slightly larger $(1.0 \%)$ than the in-plane lattice constant of wurtzite AlN of $3.127 \AA$. The corresponding average lattice constant in $\mathrm{MgGeN}_{2}$ is $3.267 \AA$, which is 
slightly larger (1.5\%) than what is obtained within the PBE approximation for wurtzite GaN (3.218 $\AA$ ). Furthermore, by using the wurtzite equivalent lattice constant $a_{w}$, it is possible to define a deviation from hexagonality in the $x y$-plane of the orthorhombic structures by evaluating $|a / \sqrt{3}-b / 2| / a_{w}$. In the case of perfect hexagonality, this expression will be zero, and the larger the value becomes the more the orthorhombic structure deviates from the hexagonal structure in the $x y$-plane. In $\mathrm{MgSiN}_{2}$, this deviation is $5.7 \%$, while it is $3.8 \%$ in $\mathrm{MgGeN}_{2} . \mathrm{MgSiN}_{2}$, therefore, deviates more from the hexagonal form of the wurtzite crystal structure compared to $\mathrm{MgGeN}_{2}$.

Regarding the out-of-plane lattice constants along the $z$-direction, we find the $c$ lattice constant in $\mathrm{MgSiN}_{2}$ to be slightly larger than the $c$ lattice constant in AlN, $5.028 \AA$ versus $5.013 \AA$, respectively. In $\mathrm{MgGeN}_{2}$, the $c$ lattice constant is larger and more similar in size to the corresponding lattice constant in $\mathrm{GaN}, 5.223 \AA$ in $\mathrm{MgGeN}_{2}$ versus $5.243 \AA$ in $\mathrm{GaN}$. It is therefore clear that the relative volume and other structural properties increase going from $\mathrm{AlN}$ to $\mathrm{MgSiN}_{2}$ to $\mathrm{MgGeN}_{2}$, and, furthermore, while the difference between AlN and $\mathrm{MgSiN}_{2}$ is rather small, $\mathrm{MgGeN}_{2}$ is structurally more similar to wurtzite GaN.

Due to the overall structural similarity between $\mathrm{MgSiN}_{2}$ and AlN, as well as between $\mathrm{MgGeN}_{2}$ and GaN, it should be possible to grow epitaxial structures along the $c$ axis composed of $\mathrm{MgSiN}_{2}$ and $\mathrm{AlN}$, as well as structures composed of $\mathrm{MgGeN}_{2}$ and $\mathrm{GaN}$, without significant strain. It is also possible that a small amount of $\mathrm{Si}$ substitution on the $\mathrm{Ge}$ sites in $\mathrm{MgGeN}_{2}$ will create a situation where the lattice mismatch with $\mathrm{GaN}$ vanishes which will be very beneficial when considering the epitaxial growth of heterostructures.

\section{DIELECTRIC TENSORS AND BORN EFFECTIVE CHARGES}

The dielectric tensors of $\mathrm{MgSiN}_{2}, \mathrm{MgGeN}_{2}$ and $\mathrm{AlN}$ are shown in Table III. In the case of $\mathrm{MgSiN}_{2}$ and $\mathrm{MgGeN}_{2}$, we find the dielectric tensor to be anisotropic, with the largest component along the $c$-axis and the smallest component along the $b$-axis in the case of $\mathrm{MgSiN}_{2}$. For $\mathrm{MgGeN}_{2}$, the largest component is along the $a$-axis, while the smallest component is along the $b$-axis. In both these cases, the $a$ - and $c$-components are similar in size, while the $b$-component is much smaller. The absolute values of the dielectric tensor are larger in $\mathrm{MgGeN}_{2}$ compared to $\mathrm{MgSiN}_{2}$ which is related to the reduced band gap in $\mathrm{MgGeN}_{2}$. When comparing the dielectric tensor of $\mathrm{MgSiN}_{2}$ to wurtzite AlN, we find both

TABLE III. The dielectric tensor, $\boldsymbol{\varepsilon}^{\infty}, \mathrm{MgSiN}_{2}$ and wurtzite AlN. Note that the $\boldsymbol{\varepsilon}_{x x}$ component for AlN is the in-plane dielectric constant, while the $\boldsymbol{\varepsilon}_{z z}$ component is the out-of-plane dielectric constant along the $c$-axis.

\begin{tabular}{lccc}
\hline \hline & $\boldsymbol{\varepsilon}_{x x}$ & $\boldsymbol{\varepsilon}_{y y}$ & $\boldsymbol{\varepsilon}_{z z}$ \\
\hline $\mathrm{MgSiN}_{2}$ & 4.37 & 4.29 & 4.42 \\
$\mathrm{MgGeN}_{2}$ & 5.12 & 4.88 & 5.09 \\
$\mathrm{AlN}$ & 4.47 & $\ldots$ & 4.70 \\
$\mathrm{AlN}^{35}$ & 5.17 & $\ldots$ & 5.36 \\
$\mathrm{AlN}^{36}$ & 4.38 & $\ldots$ & 4.61 \\
\hline \hline
\end{tabular}

TABLE IV. The Born effective charge tensor $\boldsymbol{Z}^{*}$ for $\mathrm{MgSiN}_{2}$. The \pm and $\mp$ symbols used for the off-diagonal components represent that each off-diagonal component can be both positive and negative for atoms of the same species, see text for details.

\begin{tabular}{lcccc}
\hline \hline & $\mathrm{Mg}$ & $\mathrm{Si}$ & $\mathrm{N}(1)$ & $\mathrm{N}(2)$ \\
\hline $\boldsymbol{Z}_{x x}^{*}$ & 1.92 & 3.17 & -2.04 & -3.04 \\
$\boldsymbol{Z}_{x y}^{*}$ & \pm 0.04 & $\mp 0.19$ & $\mp 0.11$ & \pm 0.47 \\
$\boldsymbol{Z}_{y x}^{*}$ & $\mp 0.04$ & \pm 0.19 & $\mp 0.30$ & \pm 0.51 \\
$\boldsymbol{Z}_{y y}^{*}$ & 1.95 & 3.02 & -2.50 & -2.47 \\
$\boldsymbol{Z}_{y z}^{*}$ & \pm 0.01 & $\mp 0.20$ & \pm 0.49 & $\mp 0.15$ \\
$\boldsymbol{Z}_{z y}^{*}$ & $\mp 0.04$ & \pm 0.23 & \pm 0.49 & $\mp 0.13$ \\
$\boldsymbol{Z}_{z z}^{*}$ & 2.08 & 3.12 & -3.04 & -2.16 \\
$\boldsymbol{Z}_{x z}^{*}$ & $\mp 0.02$ & $\mp 0.05$ & \pm 0.17 & $\mp 0.10$ \\
$\boldsymbol{Z}_{z x}^{*}$ & $\mp 0.03$ & \pm 0.09 & \pm 0.19 & $\mp 0.04$ \\
\hline \hline
\end{tabular}

TABLE V. The Born effective charge tensor $Z^{*}$ for $\mathrm{MgGeN}_{2}$. The \pm and $\mp$ symbols used for the off-diagonal components represent that each offdiagonal component can be both positive and negative for atoms of the same species, see text for details.

\begin{tabular}{lcccc}
\hline \hline & $\mathrm{Mg}$ & $\mathrm{Ge}$ & $\mathrm{N}(1)$ & $\mathrm{N}(2)$ \\
\hline $\boldsymbol{Z}_{x x}^{*}$ & 1.86 & 3.18 & -2.07 & -2.98 \\
$\boldsymbol{Z}_{x y}^{*}$ & \pm 0.16 & $\mp 0.44$ & $\mp 0.04$ & \pm 0.34 \\
$\boldsymbol{Z}_{y x}^{*}$ & $\mp 0.07$ & \pm 0.31 & $\mp 0.33$ & \pm 0.47 \\
$\boldsymbol{Z}_{y y}^{*}$ & 1.97 & 2.96 & -2.48 & -2.45 \\
$\boldsymbol{Z}_{y z}^{*}$ & \pm 0.08 & $\mp 0.44$ & \pm 0.41 & $\mp 0.11$ \\
$\boldsymbol{Z}_{z y}^{*}$ & $\mp 0.11$ & \pm 0.38 & \pm 0.42 & $\mp 0.10$ \\
$\boldsymbol{Z}_{z z}^{*}$ & 2.02 & 3.15 & -2.97 & -2.21 \\
$\boldsymbol{Z}_{x z}^{*}$ & \pm 0.01 & $\mp 0.09$ & \pm 0.14 & $\mp 0.10$ \\
$\boldsymbol{Z}_{z x}^{*}$ & $\mp 0.08$ & \pm 0.19 & \pm 0.16 & $\mp 0.02$ \\
\hline \hline
\end{tabular}

the $a$ - and $b$-components to be smaller than the in-plane dielectric constant in AlN. Furthermore, we also find the $c$-component in $\mathrm{MgSiN}_{2}$ to be smaller than the out-of-plane component in AlN. In Table III, we also show the dielectric tensors of AlN obtained in earlier computational studies, and our calculations are in good agreement with these previous studies.

The Born effective charge tensors in $\mathrm{MgSiN}_{2}, \mathrm{MgGeN}_{2}$ and AlN are shown in Tables IV-VI. These charges are related to lattice vibrations and also directly related to the LOTO splitting, as can be seen in Eq. (1), where larger values for the Born effective charges provide a larger LO-TO splitting, especially in combination with smaller values of the dielectric tensor components. In the case of $\mathrm{MgSiN}_{2}$ and $\mathrm{MgGeN}_{2}$, the Born effective charge tensors are anisotropic with nonnegligible off-diagonal elements. We note that the effective charges are sensitive to structural details. In a cubic environment, for example, the effective charges are diagonal and isotropic. However, for non-cubic structures and especially for

TABLE VI. The Born effective charge tensor $Z^{*}$ for wurtzite AlN.

\begin{tabular}{lccc}
\hline \hline & $\boldsymbol{Z}_{x x}^{*}$ & $\boldsymbol{Z}_{y y}^{*}$ & $\boldsymbol{Z}_{z z}^{*}$ \\
\hline $\mathrm{Al}(\mathrm{N})$ & 2.53 & 2.53 & 2.69 \\
$\mathrm{Al}(\mathrm{N})^{35}$ & 2.70 & 2.70 & 2.85 \\
$\mathrm{Al}(\mathrm{N})^{36}$ & 2.63 & 2.53 & 2.69 \\
\hline \hline
\end{tabular}


systems with complex local environments, non-diagonal and anisotropic effective charges are not surprising. ${ }^{37}$ We note that the diagonal elements of the effective charge tensors are similar in $\mathrm{MgSiN}_{2}$ and $\mathrm{MgGeN}_{2}$, where the largest effective charges are found for the $\mathrm{Si}$ and $\mathrm{Ge}$ atoms in each system. We note that the average of the cation (and anion) diagonal components in $\mathrm{MgSiN}_{2}$, e.g., $\boldsymbol{Z}_{x x}^{*}=\left(\boldsymbol{Z}_{x x}^{*}(\mathrm{Mg})+\boldsymbol{Z}_{x x}^{*}(\mathrm{Si})\right) / 2$ $=(1.92+3.17) / 2=2.545$, is very similar to the effective charges in AlN shown in Table VI.

Regarding the off-diagonal components, we note that these change sign even for atoms of same atomic species such that each off-diagonal component of the tensor is both positive and negative for two atoms of the same species in the unit cell. When averaged over all atoms of the same species in the unit cell, the off-diagonal components become zero and the Born effective charges therefore fulfill the sumrule $\sum_{s} \boldsymbol{Z}_{s, i j}^{*}=0$, where $s$ represents the atoms in the unit cell. The sum-rule reflects the fact that when summed over all atoms in the system, each component of the Born effective charge tensor should vanish.

\section{V. Г-POINT MODES AND RAMAN SPECTRA}

We begin the discussion of the lattice dynamics by analysing the vibrations at the Brillouin zone center, i.e., at the $\Gamma$-point. The applicable point group for the orthorhombic Pna2 $2_{1}$ structure is $C_{2 v}$, and it has four irreducible representations $\mathrm{A}_{1}, \mathrm{~A}_{2}, \mathrm{~B}_{1}$ and $\mathrm{B}_{2}$. We note that $\mathrm{A}_{1}, \mathrm{~B}_{1}$ and $\mathrm{B}_{2}$ correspond to modes with the same symmetry as a vector along the $z, x$ and $y$ crystal directions, respectively. These are the modes that are subject to a LO-TO splitting according to Eq. (1), while the $\mathrm{A}_{2}$ modes are unaffected. Since the orthorhombic unit cell contains 16 atoms, there are 48 modes (12 for each symmetry), of which 3 are acoustic with zero frequency at the $\Gamma$-point. The zero frequency modes are of $A_{1}, B_{1}$ and $B_{2}$ symmetry. The remaining modes are all Raman active. Due to the LO-TO splitting, the frequency of the remaining $11 \mathrm{~A}_{1}, 11 \mathrm{~B}_{1}$ and $11 \mathrm{~B}_{2}$ modes will depend on the direction from which the $\Gamma$-point is approached. That is, as an example, a static field in the $z$ direction will affect the $\mathrm{A}_{1}$ modes, and therefore, if the $\Gamma$-point is approached along the [001] direction, i.e., along the $z$-axis, the $\mathrm{A}_{1}^{\mathrm{LO}}$ mode will be obtained. If the $\Gamma$-point is approached along $x$ or $y$ directions, the $\mathrm{A}_{1}^{\mathrm{TO}}$ mode will be obtained. In Figs. 3 and 4, we show the calculated frequencies of the $\Gamma$-point modes for both $\mathrm{MgSiN}_{2}$ and $\mathrm{MgGeN}_{2}$. These frequencies are also presented in Tables VII and VIII. For both systems, the highest frequency mode along [001], [010] and [100] are $A_{1}^{\mathrm{LO}}, \mathrm{B}_{2}^{\mathrm{LO}}$ and $\mathrm{B}_{1}^{\mathrm{LO}}$ modes, respectively. In the case of $\mathrm{MgSiN}_{2}$, the highest frequency is found to be $996.4 \mathrm{~cm}^{-1}$ for the $\mathrm{A}_{1}^{\mathrm{LO}}$ mode, which is more than $100 \mathrm{~cm}^{-1}$ higher than what we find for the $\mathrm{E}_{1}^{\mathrm{LO}}$ mode $\left(885.4 \mathrm{~cm}^{-1}\right)$ in wurtzite AlN. The $\mathrm{A}_{1}^{\mathrm{LO}}$ mode in $\mathrm{MgGeN}_{2}$ is $784.0 \mathrm{~cm}^{-1}$. We note that in the $\mathrm{Zn}$-based II-IV nitrides $\mathrm{ZnSiN}_{2}, \mathrm{ZnGeN}_{2}$ and $\mathrm{ZnSnN}_{2}$, the highest frequencies are $980.0 \mathrm{~cm}^{-1}\left(\mathrm{~A}_{1}^{\mathrm{LO}}\right),{ }^{38} 859.8 \mathrm{~cm}^{-1}$ $\left(\mathrm{B}_{2}^{\mathrm{LO}}\right),{ }^{39}$ and $739 \mathrm{~cm}^{-1}\left(\mathrm{~B}_{2}^{\mathrm{LO}}\right),{ }^{40}$ respectively. We therefore note that as the mass of group II and IV elements increases the modes become softer and that both $\mathrm{MgSiN}_{2}$ and $\mathrm{ZnSiN}_{2}$ have modes with significantly higher frequency than found in AlN.

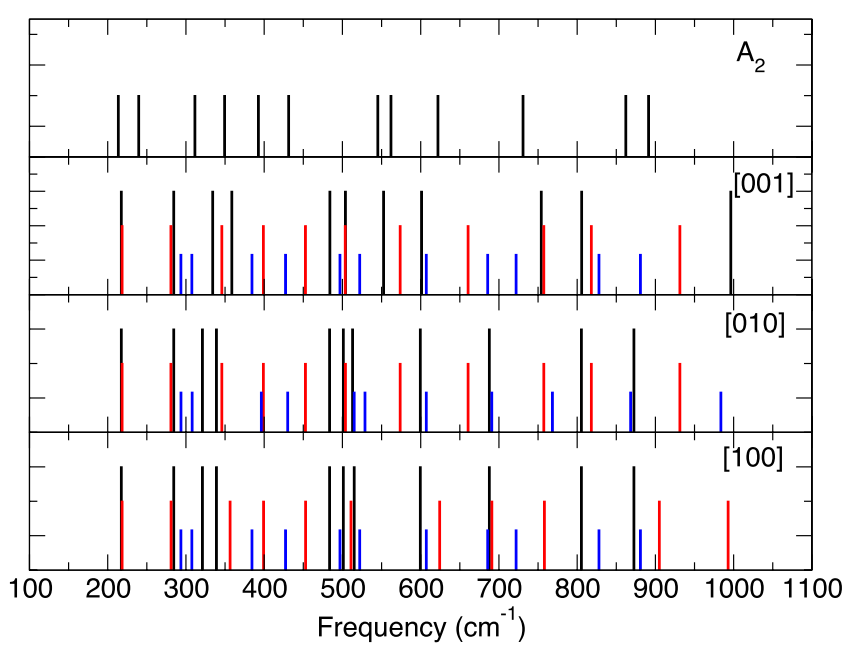

FIG. 3. Calculated phonon modes at the $\Gamma$-point of $\operatorname{MgSiN}_{2}$. The top panel contains the $\mathrm{A}_{2}$ modes. The other panels contain the $\mathrm{A}_{1}, \mathrm{~B}_{1}$ and $\mathrm{B}_{2}$ modes for $\boldsymbol{q} \rightarrow 0$ along [001], [010], and [100]. $\mathrm{A}_{1}, \mathrm{~B}_{1}$, and $\mathrm{B}_{2}$ modes are shown using (tall) black, red, and (short) blue bars, respectively.

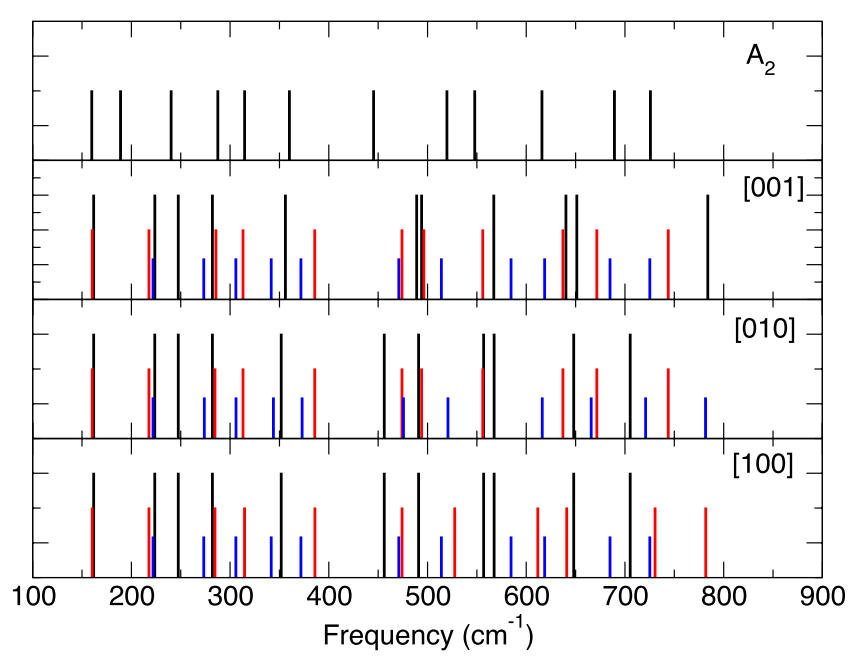

FIG. 4. Calculated phonon modes at the $\Gamma$-point of $\mathrm{MgGeN}_{2}$. The top panel contains the $\mathrm{A}_{2}$ modes. The other panels contain the $\mathrm{A}_{1}, \mathrm{~B}_{1}$ and $\mathrm{B}_{2}$ modes for $\boldsymbol{q} \rightarrow 0$ along [001], [010] and [100]. $\mathrm{A}_{1}, \mathrm{~B}_{1}$, and $\mathrm{B}_{2}$ modes are shown using (tall) black, red, and (short) blue bars, respectively.

TABLE VII. Calculated phonon modes at the $\Gamma$-point of $\mathrm{MgSiN}_{2}$ sorted according to their symmetry.

\begin{tabular}{lllllll}
\hline \hline $\mathrm{A}_{1}^{\mathrm{TO}}$ & $\mathrm{A}_{1}^{\mathrm{LO}}$ & $\mathrm{B}_{1}^{\mathrm{TO}}$ & $\mathrm{B}_{1}^{\mathrm{LO}}$ & $\mathrm{B}_{2}^{\mathrm{TO}}$ & $\mathrm{B}_{2}^{\mathrm{LO}}$ & $\mathrm{A}_{2}$ \\
\hline 217.4 & 217.4 & 218.4 & 218.4 & 293.5 & 293.6 & 213.6 \\
284.4 & 284.4 & 280.8 & 281.0 & 307.5 & 307.9 & 239.6 \\
321.1 & 334.2 & 345.9 & 356.3 & 384.3 & 396.3 & 311.4 \\
339.0 & 358.6 & 398.9 & 399.2 & 427.3 & 430.0 & 349.4 \\
483.6 & 483.9 & 452.7 & 452.8 & 496.8 & 515.1 & 392.6 \\
501.1 & 503.9 & 503.6 & 510.9 & 522.1 & 528.8 & 431.2 \\
515.1 & 552.6 & 573.8 & 624.3 & 607.2 & 607.2 & 545.2 \\
599.5 & 601.1 & 660.6 & 691.0 & 685.6 & 690.8 & 562.0 \\
687.7 & 754.1 & 757.4 & 758.2 & 721.9 & 768.4 & 622.1 \\
805.5 & 805.8 & 818.1 & 905.0 & 827.9 & 868.3 & 730.8 \\
872.6 & 996.4 & 931.3 & 993.0 & 880.9 & 983.7 & 862.2 \\
& & & & & & 891.3 \\
\hline \hline
\end{tabular}


TABLE VIII. Calculated phonon modes at the $\Gamma$-point of $\mathrm{MgGeN}_{2}$ sorted according to their symmetry.

\begin{tabular}{lcccccc}
\hline \hline $\mathrm{A}_{1}^{\mathrm{TO}}$ & $\mathrm{A}_{1}^{\mathrm{LO}}$ & $\mathrm{B}_{1}^{\mathrm{TO}}$ & $\mathrm{B}_{1}^{\mathrm{LO}}$ & $\mathrm{B}_{2}^{\mathrm{TO}}$ & $\mathrm{B}_{2}^{\mathrm{LO}}$ & $\mathrm{A}_{2}$ \\
\hline 161.8 & 161.8 & 160.3 & 160.3 & 221.9 & 221.9 & 159.9 \\
223.7 & 223.8 & 217.8 & 217.8 & 273.3 & 273.9 & 189.8 \\
247.5 & 247.5 & 285.6 & 284.7 & 305.8 & 306.0 & 240.2 \\
282.1 & 282.1 & 313.1 & 314.7 & 341.6 & 343.9 & 287.6 \\
351.8 & 356.0 & 385.7 & 385.9 & 371.8 & 372.9 & 314.7 \\
456.2 & 489.0 & 474.1 & 474.2 & 470.9 & 475.6 & 360.1 \\
490.9 & 494.0 & 496.3 & 527.5 & 514.0 & 520.7 & 445.4 \\
556.8 & 567.2 & 556.0 & 611.8 & 584.6 & 616.3 & 519.7 \\
567.5 & 640.2 & 637.2 & 641.0 & 618.7 & 665.8 & 547.9 \\
648.1 & 651.2 & 671.5 & 730.5 & 684.9 & 720.9 & 615.9 \\
705.3 & 784.0 & 743.9 & 781.8 & 725.2 & 781.6 & 689.4 \\
& & & & & & 725.8 \\
\hline \hline
\end{tabular}

Figure 5 shows the results of Raman measurements of a powder sample of $\mathrm{MgSiN}_{2}$, where the principal $\mathrm{MgSiN}_{2}$ peaks are highlighted with their frequency. We note that even though there exist 45 Raman active modes according to the symmetry analysis of $\mathrm{MgSiN}_{2}$, all of them might not show up in Raman measurements since the intensity of some of the peaks might be very low. In addition to the principal peaks, several peaks indicate the presence of free $\mathrm{Si}$ in the powder, in keeping with observations made for the $\mathrm{Mg} / \mathrm{Si}_{3} \mathrm{~N}_{4}$ synthesis route by Bruls et al. ${ }^{41}$ Other impurity peaks corresponding to $\mathrm{MgO}$ are also present. The impurity peaks were assigned by referring to Raman spectra obtained for the impurity compounds on the same Raman system. As seen in Fig. 5, there are 12 peaks derived solely from $\mathrm{MgSiN}_{2}$ at 225, 329, 354, 408, 567, 623, 712, 751, $833,893,917$, and $1026 \mathrm{~cm}^{-1}$. These frequencies correspond agreeably with the calculated frequencies of Fig. 3 and Table VII. Especially, these Raman results support the finding that the highest frequency mode in $\mathrm{MgSiN}_{2}$ has a frequency that is significantly higher than the highest frequency found in AIN. Furthermore, we find it to be likely that impurity peaks mask the presence of additional $\mathrm{MgSiN}_{2}$ Raman peaks.

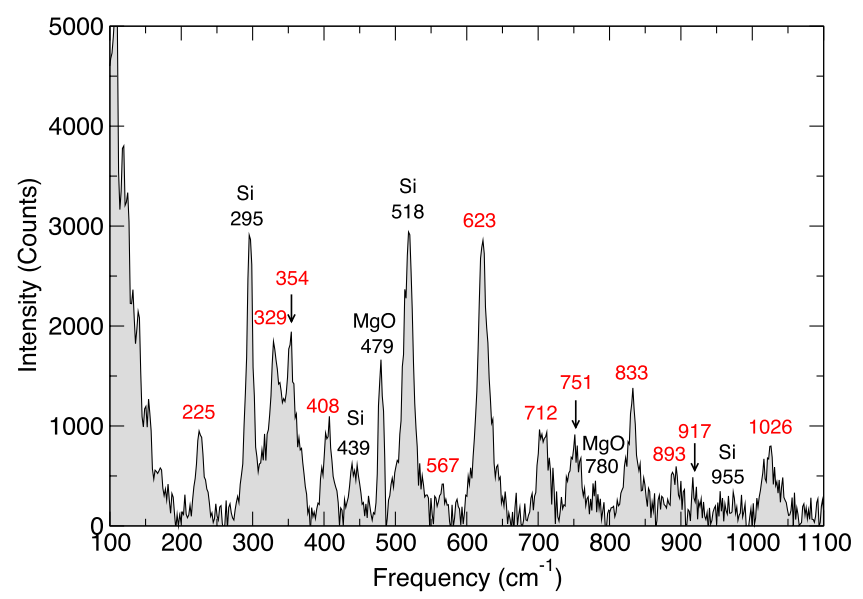

FIG. 5. Measured Raman spectra of a $\mathrm{MgSiN}_{2}$ powder sample. Impurity peaks and assigned frequencies are shown in black. Unique peaks due to $\mathrm{MgSiN}_{2}$ are shown in red.

\section{LATTICE DYNAMICS}

Figure 6 shows the phonon dispersions along high symmetry directions in the Brillouin zone of AlN in the wurtzite structure. The lattice dynamics of AlN has already been discussed thoroughly by, for example, Bungaro et al., ${ }^{35}$ and we will summarise only some general features of relevance when comparing to the more complex Mg-IV- $\mathrm{N}_{2}$ systems. The wurtzite unit cell contains 4 atoms and therefore there are 12 modes in the vibrational spectrum. There is a separation centered around $560 \mathrm{~cm}^{-1}$ between higher optical modes and the lower energy modes. As was previously mentioned, the highest optical frequency is $885.4 \mathrm{~cm}^{-1}$ for the LO mode with $\mathrm{E}_{1}$ symmetry, which is slightly lower than the experimental frequency ${ }^{42}$ of $916 \mathrm{~cm}^{-1}$. We note that the underestimation obtained in the calculations compared to the available Raman frequencies in AIN, shown in Fig. 6, is likely due to the use of the PBE approximation, which tends to underestimate the phonon frequencies found experimentally due to the general underbinding obtained when using this approximation.

$\mathrm{By}$ replacing two $\mathrm{Al}$ atoms for one $\mathrm{Mg}$ and one $\mathrm{Si}$ or $\mathrm{Ge}$ atom, the size of the system is doubled, but the unit cells for $\mathrm{MgSiN}_{2}$ and $\mathrm{MgGeN}_{2}$ contain 4 formula units (f.u.), as discussed in Sec. III, and the number of phonon modes is therefore 48. As a consequence, the phonon dispersions in $\mathrm{MgSiN}_{2}$ and $\mathrm{MgGeN}_{2}$ shown in Figs. 7 and 8 are much more complex than the dispersions in AlN. In the case of $\mathrm{MgSiN}_{2}$, shown in Fig. 7, we find that the separation found in AlN that centered around $560 \mathrm{~cm}^{-1}$ has vanished. Instead, there is a small gap in the vibrations slightly below $800 \mathrm{~cm}^{-1}$. In addition, there are a number of modes with small dispersion just above $800 \mathrm{~cm}^{-1}$ that give rise to a tall peak in the phonon density of states shown in Fig. 7. This feature is also found in $\mathrm{ZnSiN}_{2},{ }^{40}$ even though the separation between the peak and the lower frequency modes is larger in the case of $\mathrm{ZnSiN}_{2}$. There is, however, a significant difference between the vibrations in $\mathrm{MgSiN}_{2}$ and $\mathrm{ZnSiN}_{2}$ : in the latter system, there is a rather significant separation between the modes below $200 \mathrm{~cm}^{-1}$ and

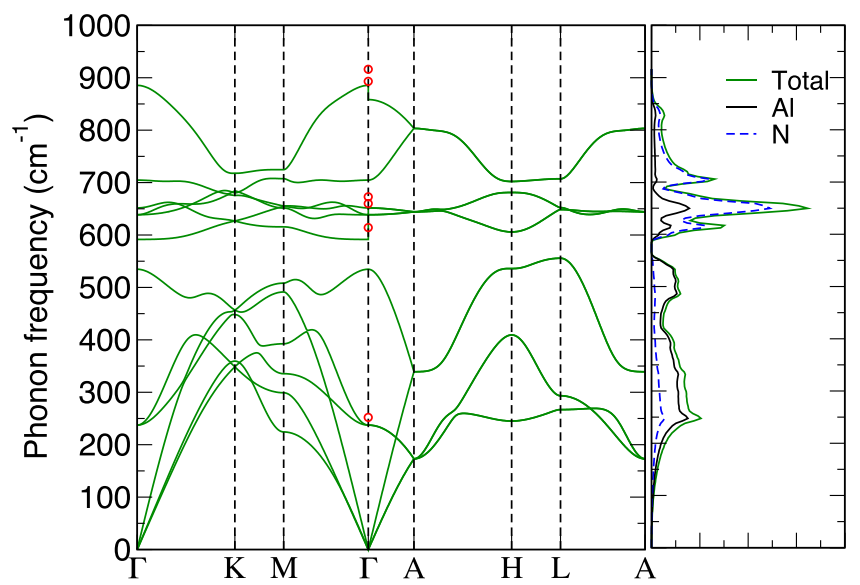

FIG. 6. The calculated phonon dispersions along high symmetry directions in the Brillouin zone (left panel) and the phonon densities of states (right panel) of wurtzite AlN. Experimental Raman frequencies ${ }^{42}$ are shown using (red) circles. The phonon densities of states projected onto Al and $\mathrm{N}$ atoms are also shown. 


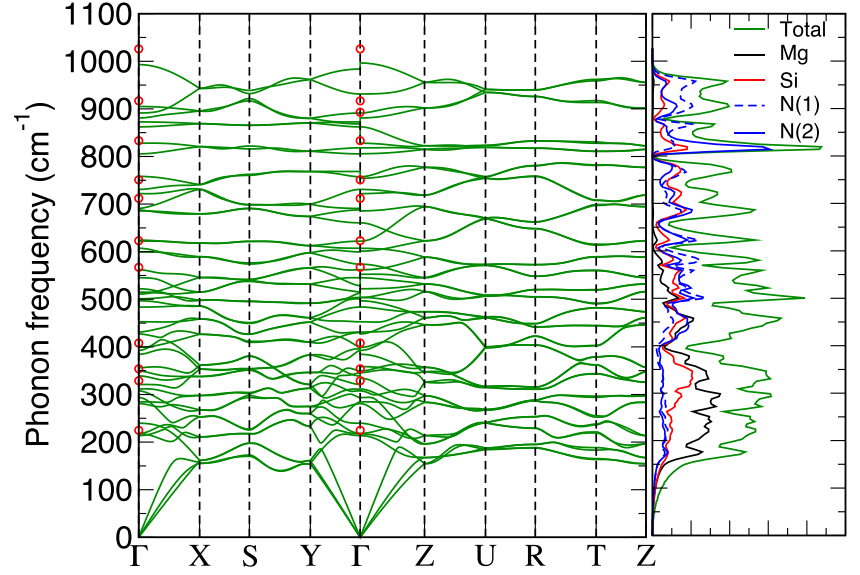

FIG. 7. The calculated phonon dispersions along high symmetry directions in the Brillouin zone (left panel) and the phonon densities of states (right panel) of orthorhombic $\mathrm{MgSiN}_{2}$. Experimental Raman frequencies are shown using (red) circles. The phonon densities of states projected onto $\mathrm{Mg}$, $\mathrm{Si}, \mathrm{N}(1)$, and $\mathrm{N}(2)$ atoms are also shown.

the higher frequency optical modes. ${ }^{40} \mathrm{In} \mathrm{MgSiN}_{2}$, these two regions have mixed into a single region.

When $\mathrm{Si}$ is substituted for the heavier $\mathrm{Ge}$, the lattice vibrations become softer in general, as shown in Fig. 8, and for $\mathrm{MgGeN}_{2}$, the highest frequency is $784.0 \mathrm{~cm}^{-1}$. Compared to $\mathrm{MgSiN}_{2}$, the lattice vibrations in $\mathrm{MgGeN}_{2}$ are more structured with a clear separation between high frequency optical modes and lower frequency modes between $400 \mathrm{~cm}^{-1}$ and $440 \mathrm{~cm}^{-1}$. Furthermore, there are two additional gaps, where the lower gap is located at $530 \mathrm{~cm}^{-1}$ and the upper gap is located at about $710 \mathrm{~cm}^{-1}$.

In Figs. 6-8, we also present the phonon density of states of $\mathrm{AlN}, \mathrm{MgSiN}_{2}$ and $\mathrm{MgGeN}_{2}$ projected onto each atomic species. In AlN, it is clear that cation vibrations dominate at low frequencies and anion vibrations at high frequencies. The same is also true for $\mathrm{MgSiN}_{2}$ and $\mathrm{MgGeN}_{2}$, where in both cases the phonon density of states is dominated by cation vibrations up to about $400 \mathrm{~cm}^{-1}$. Above this point, the vibrations are more dominated by nitrogen vibrations.

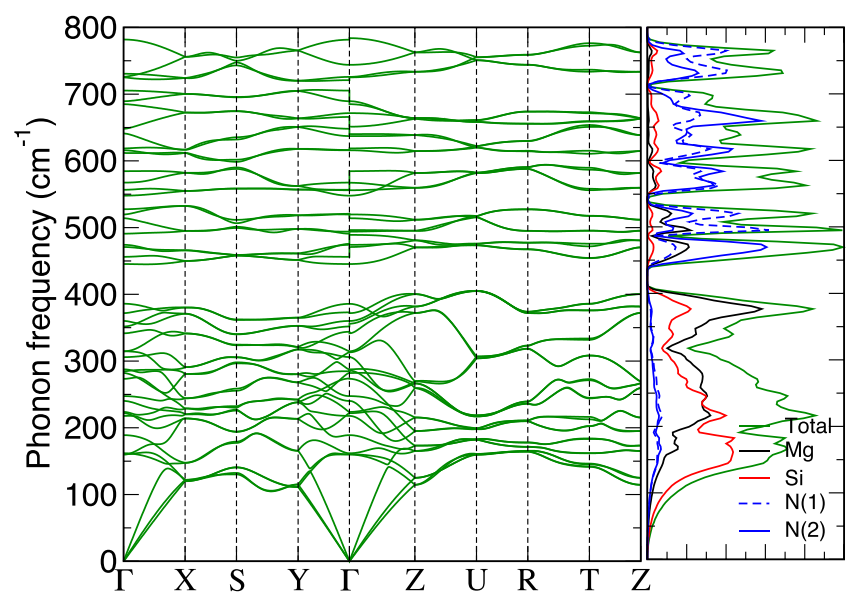

FIG. 8. The calculated phonon dispersions along high symmetry directions in the Brillouin zone (left panel) and the phonon density of states (right panel) of orthorhombic $\mathrm{MgGeN}_{2}$. The phonon densities of states projected onto $\mathrm{Mg}, \mathrm{Ge}, \mathrm{N}(1)$, and $\mathrm{N}(2)$ atoms are also shown.

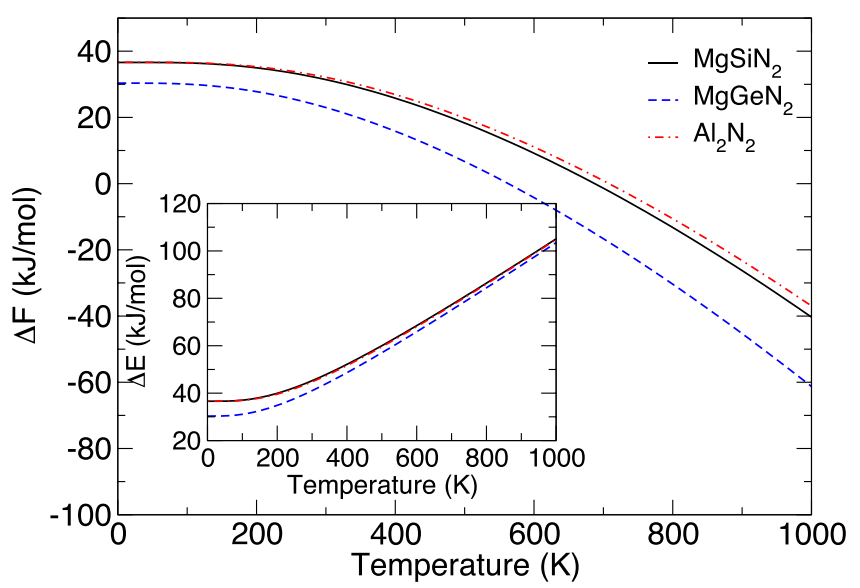

FIG. 9. The calculated Helmholtz free energy and total energy (inset) due to lattice vibrations of $\mathrm{MgSiN}_{2}, \mathrm{MgGeN}_{2}$, and $\mathrm{AlN}$.

\section{THERMODYNAMIC PROPERTIES}

The contribution of the phonons to thermodynamic properties, such as the Helmholtz free energy $F$, the total energy $E$, the specific heat $C_{v}=(\partial E / \partial T)_{v}$ and the entropy $S=(E-F) / T$, is expressed through the partition function

$$
Z=\Pi_{\lambda} \frac{e^{-\beta \hbar \omega_{\lambda} / 2}}{1-e^{-\beta \hbar \omega_{\lambda}}}
$$

where $\lambda$ is a combined summation index over both modes and q-points and $\omega_{\lambda}$ is the vibrational frequency of mode $\nu$ at q-point $\boldsymbol{q}$. The Helmholtz free energy is given by

$$
F=-\frac{1}{\beta} \ln Z=\sum_{\lambda}\left[\frac{\hbar \omega_{\lambda}}{2}+\frac{1}{\beta} \ln \left(1-\exp \left(-\beta \hbar \omega_{\lambda}\right)\right)\right],
$$

and the total energy is given by

$$
E=-\frac{\partial \ln Z}{\partial \beta}=\sum_{\lambda} \hbar \omega_{\lambda}\left[\frac{1}{2}+\frac{1}{\exp \left(\beta \hbar \omega_{\lambda}\right)-1}\right] .
$$

In Figs. 9 and 10, we show the calculated free energies, total energies, heat capacities and entropies of $\mathrm{MgSiN}_{2}$, $\mathrm{MgGeN}_{2}$ and AlN. Note that in order to facilitate an easier comparison between AIN and the two II-IV nitrides, we have

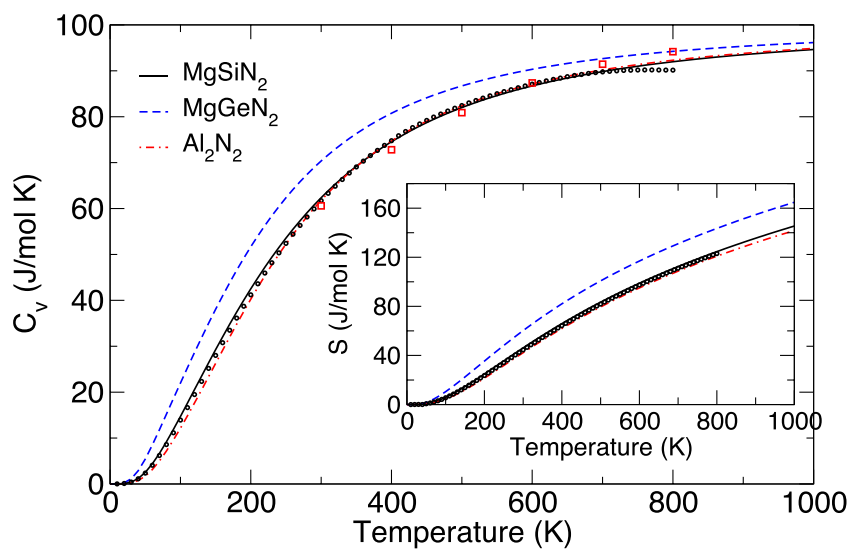

FIG. 10. The specific heat at constant volume and entropy (inset) of $\mathrm{MgSiN}_{2}, \mathrm{MgGeN}_{2}$ and AlN. Experimental data for $\mathrm{MgSiN}_{2}$ (black points) and AlN (red squares) are taken from Ref. 19. 
plotted the AlN-related curves as if the system is twice as large, i.e., we show the free energy, total energy, heat capacity and entropy for $\mathrm{Al}_{2} \mathrm{~N}_{2}=2$. AlN, instead of AlN. Otherwise, all properties shown in Figs. 9 and 10 are about twice as large for $\mathrm{MgSiN}_{2}$ than for AlN.

The zero point energies, i.e., the total energy at $T=0 \mathrm{~K}$ for $\mathrm{MgSiN}_{2}, \mathrm{MgGeN}_{2}$ and $\mathrm{AlN}$ is $36.6,30.3$ and $18.3 \mathrm{~kJ} / \mathrm{mol}$, respectively, or $0.190,0.157$ and $0.190 \mathrm{eV}$ per nitrogen atom, respectively. In comparison, the calculated zero point energy in $\mathrm{ZnSiN}_{2}$ is $34.0 \mathrm{~kJ} / \mathrm{mol}$ or $0.176 \mathrm{eV}$ per nitrogen atom, ${ }^{40}$ which is slightly less than what is found for $\mathrm{MgSiN}_{2}$ but slightly higher than what is found for $\mathrm{MgGeN}_{2}$. This is expected due to the differences in the mass between $\mathrm{Mg}, \mathrm{Si}$, $\mathrm{Ge}$ and $\mathrm{Zn}$, where $\mathrm{Zn}$ is heavier than $\mathrm{Mg}$, but Ge is heavier than $\mathrm{Si}$ and $\mathrm{Zn}$, which makes $\mathrm{MgGeN}_{2}$ a heavier system than both $\mathrm{MgSiN}_{2}$ and $\mathrm{ZnSiN}_{2}$.

The curves for $\mathrm{AlN}$ and $\mathrm{MgSiN}_{2}$ follow each other closely in Figs. 9 and 10. The free and total energies of $\mathrm{MgGeN}_{2}$ are smaller than those of $\mathrm{MgSiN}_{2}$; the difference between the free energy of the two compounds increases with temperature, but in contrast, the difference in total energy becomes smaller with increasing temperature. In Fig. 10 , it is clear that both the specific heat and the entropy are larger in $\mathrm{MgGeN}_{2}$ compared to $\mathrm{MgSiN}_{2}$. This behaviour is identical to what is observed for $\mathrm{Zn}-\mathrm{IV}-\mathrm{N}_{2}$, where the free and total energies decrease from $\mathrm{ZnSiN}_{2}$ to $\mathrm{ZnGeN}_{2}$ to $\mathrm{ZnSnN}_{2}$, while the heat capacity and entropy increase from $\mathrm{ZnSiN}_{2}$ to $\mathrm{ZnGeN}_{2}$ to $\mathrm{ZnSnN}_{2}{ }^{40}$

When comparing our calculated thermodynamic properties to available experimental studies, we find a very good agreement regarding the vibrational entropy in the case of $\mathrm{MgSiN}_{2}$, as is shown in Fig. 10. In Fig. 10, we also show the experimental heat capacity at constant pressure $\left(C_{p}\right)$, which is related to the heat capacity at constant volume $\left(C_{v}\right)$ by

$$
C_{p}-C_{v}=9 \alpha^{2} \frac{V_{m} T}{\beta_{T}},
$$

where $\alpha$ is the thermal expansion, $V_{m}$ is the molar volume and $\beta_{T}$ is the isothermal compressibility. The difference between $C_{p}$ and $C_{v}$ is a continuously increasing function of temperature. By using experimental values for the thermal expansion, volume and compressibility, Bruls et al. ${ }^{19}$ have shown that the difference in the left hand side of Eq. (5) at $800 \mathrm{~K}$ is $1.2 \mathrm{~J} / \mathrm{mol} / \mathrm{K}$, i.e., a relative difference of about $1.3 \%$, which was well inside the accuracy of the measurement. Therefore, for sufficiently low temperatures, the heat capacities at constant pressure and constant volume are approximately the same for $\mathrm{MgSiN}_{2}$, and a comparison between experimental values for the heat capacity at constant pressure and the theoretical heat capacity at constant volume is relevant. As can be seen in Fig. 10, the experimental and theoretical curves follow each other closely up to about $700 \mathrm{~K}$. We note that the calculated curve is slightly higher than the experimental curve for $\mathrm{T} \leq 400 \mathrm{~K}$, after which the experimental curve is higher until this curve flattens at about $700 \mathrm{~K}$.

\section{SUMMARY AND CONCLUSIONS}

We have performed density functional calculations of the structural and lattice dynamical properties of $\mathrm{MgSiN}_{2}$ and $\mathrm{MgGeN}_{2}$. Our calculations are in very good agreement with available experimental results, especially regarding the structural properties, but also regarding thermodynamic properties, i.e., heat capacity and entropy, in the case of $\mathrm{MgSiN}_{2}$. We find that $\mathrm{MgSiN}_{2}$ is structurally very similar to wurtzite AlN, with very good in-plane lattice matching, while $\mathrm{MgGeN}_{2}$ has a larger volume and is more similar to wurtzite GaN. The phonon dispersions in $\mathrm{MgSiN}_{2}$ and $\mathrm{MgGeN}_{2}$ are much more complex than in AlN. We also find that the highest optical mode is about $100 \mathrm{~cm}^{-1}$ higher in energy in $\mathrm{MgSiN}_{2}$ compared to $\mathrm{AlN}$, and that the lattice vibrations in $\mathrm{MgGeN}_{2}$ are softer than in both $\mathrm{MgSiN}_{2}$ and AlN. The free energy and the total energy due to lattice vibrations are smaller in $\mathrm{MgGeN}_{2}$ than in $\mathrm{MgSiN}_{2}$, while the opposite is the case for the heat capacity and the entropy. When comparing the thermodynamic properties, i.e., free energy, total energy, heat capacity and entropy, of $\mathrm{MgSiN}_{2}$ and AlN, we find that the curves as a function of temperature follow each other closely with the difference being an approximate factor of 2 per mole. For example, the zero point energy in $\mathrm{MgSiN}_{2}$ is $36.6 \mathrm{~kJ} / \mathrm{mol}$, while it is $18.3 \mathrm{~kJ} / \mathrm{mol}$ in AlN.

\section{ACKNOWLEDGMENTS}

We acknowledge support from the Leverhulme Trust via M. A. Moram's Research Leadership Award (RL-007-2012). M. A. Moram further acknowledges support from the Royal Society through a University Research Fellowship. This work used the Imperial College high performance computing facilities and, via our membership of the UK's HEC Materials Chemistry Consortium funded by EPSRC (EP/L000202), the ARCHER UK National Supercomputing Service (http:// www.archer.ac.uk).

${ }^{1}$ S. Nakamura, S. J. Pearton, and G. Fasol, The Blue Laser Diode: The Complete Story (Springer Verlag, Berlin, Heidelberg, 2000).

${ }^{2}$ M. R. Kramers, O. B. Shchekin, R. Mueller-Mach, G. O. Mueller, L. Zhou, G. Harbers, and M. G. Craford, J. Disp. Technol. 3, 160 (2007).

${ }^{3}$ U. K. Mishra, S. Likun, T. E. Kazior, and Y. F. Wu, Proc. IEEE 96, 287 (2008).

${ }^{4}$ R. Elfrink, T. M. Kamel, M. Goedbloed, S. Matova, D. Hohlfeld, Y. van Andel, and R. van Schaijk, J. Micromech. Microeng. 19, 094005 (2009).

${ }^{5}$ M. Kneissl, T. Kolbe, C. Chua, V. Kueller, N. Lobo, J. Stellmach, A. Knauer, H. Rodriguez, S. Einfeldt, Z. Yang, N. M. Johnson, and M. Weyers, Semicond. Sci. Technol. 26, 014036 (2011).

${ }^{6}$ J. Wu, J. Appl. Phys. 106, 011101 (2009).

${ }^{7}$ T. R. Paudel and W. R. Lambrecht, Phys. Rev. B 79, 245205 (2009).

${ }^{8}$ A. Punya, T. R. Paudel, and W. R. L. Lambrecht, Phys. Status Solidi C 8, 2492 (2011).

${ }^{9}$ J. B. Quirk, M. Råsander, C. M. McGilvery, R. Palgrave, and M. A. Moram, Appl. Phys. Lett. 105, 112108 (2014).

${ }^{10}$ M. Råsander and M. A. Moram, Mater. Res. Express 3, 85902 (2016).

${ }^{11}$ A. P. Jaroenjittichai and W. R. L. Lambrecht, Phys. Rev. B 94, 125201 (2016).

${ }^{12}$ J. David, Y. Laurent, and J. Lang, Bull. Soc. Fr. Mineral. Cristallogr. 93, 153 (1970).

${ }^{13}$ C. M. Fang, R. A. de Groot, R. J. Bruls, H. T. Hintzen, and G. de With, J. Phys.: Condens. Matter 11, 4833 (1999).

${ }^{14}$ J. Y. Huang, L.-C. Tang, and M. H. Lee, J. Phys.: Condens. Matter 13, 10417 (2001)

${ }^{15}$ T. de Boer, T. D. Boyko, C. Braun, W. Schnick, and A. Moewes, Phys. Status Solidi RRL 9, 250 (2015).

${ }^{16}$ G. K. Gaido, G. P. Dubrovskii, and A. M. Zykov, Izv. Akad. Nauk. SSSR, Neorg. Mater. 10, 564 (1974). 
${ }^{17}$ R. J. Bruls, H. T. Hintzen, R. Metselaar, and C.-K. Loong, J. Phys. Chem. Solids 61, 1285 (2000).

${ }^{18}$ R. J. Bruls, H. T. Hintzen, and R. Metselaar, J. Euro. Ceram. Soc. 25, 767 (2005).

${ }^{19}$ R. J. Bruls, H. T. Hintzen, R. Metselaar, and J. C. van Miltenburg, J. Phys. Chem. B 102, 7871 (1998).

${ }^{20}$ F. Arab, F. A. Sahraoui, K. Haddadi, A. Bouhemadou, and L. Louail, Phase Trans. 89, 480 (2016).

${ }^{21}$ S. Pramchu, A. Punya Jaroenjittichai, and Y. Laosiritaworn, Ceramics International 43, S444-S448 (2017).

${ }^{22}$ P. E. Blöchl, Phys. Rev. B 50, 17953 (1994).

${ }^{23}$ G. Kresse and J. Furthmüller, Phys. Rev. B 54, 11169 (1996).

${ }^{24}$ G. Kresse and D. Joubert, Phys. Rev. B 59, 1758 (1999).

${ }^{25}$ J. P. Perdew, K. Burke, and M. Ernzerhof, Phys. Rev. Lett. 77, 3865 (1996).

${ }^{26}$ L. Chaput, A. Togo, I. Tanaka, and G. Hug, Phys. Rev. B 84, 094302 (2011).

${ }^{27}$ K. Parlinski, Z. Q. Li, and Y. Kawazoe, Phys. Rev. Lett. 78, 4063 (1997).

${ }^{28}$ R. M. Pick, M. H. Cohen, and R. M. Martin, Phys. Rev. B 1, 910 (1970).

${ }^{29}$ P. Giannozzi, S. Degironcoli, P. Pavone, and S. Baroni, Phys. Rev. B 43, 7231 (1991).
${ }^{30}$ X. Gonze and C. Lee, Phys. Rev. B 55, 10355 (1997).

${ }^{31}$ Y. Wang, J. J. Wang, W. Y. Wang, Z. G. Mei, S. L. Shang, L. Q. Chen, and Z. K. Liu, J. Phys.: Condens. Matter 22, 202201 (2010).

${ }^{32}$ S. Baroni and R. Resta, Phys. Rev. B 33, 7017 (1986).

${ }^{33}$ M. Gajdoš, K. Hummer, G. Kresse, J. Furthmüller, and F. Bechstedt, Phys. Rev. B 73, 045112 (2006).

${ }^{34}$ M. Råsander and M. A. Moram, J. Chem. Phys. 143, 144104 (2015).

${ }^{35}$ C. Bungaro, K. Rapcewicz, and J. Bernholc, Phys. Rev. B 61, 6720 (2000).

${ }^{36}$ K. Karch and F. Bechstedt, Phys. Rev. B 56, 7404 (1997).

${ }^{37}$ E. Cockayne and B. P. Burton, Phys. Rev. B 62, 3735 (2000).

${ }^{38}$ T. R. Paudel and W. R. L. Lambrecht, Phys. Rev. B 76, 115205 (2007).

${ }^{39}$ W. R. L. Lambrecht, E. Alldredge, and K. Kim, Phys. Rev. B 72, 155202 (2005).

${ }^{40}$ T. R. Paudel and W. R. L. Lambrecht, Phys. Rev. B 78, 115204 (2008).

${ }^{41}$ R. J. Bruls, A. A. Kudyba-Jansen, P. Gerharts, H. T. Hintzen, and R. Metselaar, J. Mater. Sci. Mater. Electron. 13, 63 (2002).

${ }^{42}$ L. E. McNeil, M. Grimsditch, and R. H. French, J. Am. Chem. Soc. 76, 1132 (1993). 